\title{
Clinical Scales as Predictors of Mortality in Patients with Acute Leukemia During Intensive Induction Therapy
}

\author{
Camila Dias Pastana, "*, Dafne Rosa Benzecry", Josy Marinho de Lima², \\ Marcos Laércio Pontes Reis ${ }^{2}$, Thiago Xavier Carneiro ${ }^{2}$ \\ ${ }^{1}$ Faculty of Medicine, University of State of Pará, Belém, Brazil \\ ${ }^{2}$ Department of Oncology and Hematology, University of State of Pará, Belém, Brazil
}

Email address:

camilapastana@ymail.com (C. D. Pastana), dafne_rosa@hotmail.com (D. R. Benzecry), josyml@gmail.com (J. M. de Lima)

marcoslaercio@gmail.com (M. L. P. Reis), thiagoxave@gmail.com (T. X. Carneiro)

*Corresponding author

\section{To cite this article:}

Camila Dias Pastana, Dafne Rosa Benzecry, Josy Marinho de Lima, Marcos Laércio Pontes Reis, Thiago Xavier Carneiro. Clinical Scales as Predictors of Mortality in Patients with Acute Leukemia During Intensive Induction Therapy. International Journal of Clinical Oncology and Cancer Research. Vol. 4, No. 2, 2019, pp. 5-9. doi: 10.11648/j.ijcocr.20190402.11

Received: March 29, 2019; Accepted: May 6, 2019; Published: June 4, 2019

\begin{abstract}
Therapy guidelines for acute leukemias (ALs) have focused on an arbitrary age cut-off as a guide for intensity of therapy. However, treatment outcomes depend on more important prognostic factors, such as performance status (PS) and the presence of comorbidities. This study aims to evaluate clinical scales as predictors of mortality in patients with acute leukemia during intensive induction therapy. This prospective cohort study included all patients diagnosed with Acute Myeloid Leukemia (AML) or Acute Lymphoblastic Leukemia (ALL) who received induction treatment at Ophir Loyola Hospital (HOL) in Belém-PA, from February 2018 to February 2019. The following scales were assessed: Eastern Cooperative Oncology Group (ECOG), Haematopoetic Cell Transplantation Comorbidity Index (HCT-CI), Cumulative Illness Rating Scale (CIRS), Charlson Comorbidity Index (CCI), Adult Comorbidity Evaluation 27 (ACE-27), Katz and Lawton scales, G8 Questionnaire and Mini Nutritional Assessment (MAN). The median age of the 40 patients included was 37 years old (range, 19-65) and sex distribution was equal. Univariate analysis showed that higher age $(\mathrm{OR}=5.74, \mathrm{p} 0.024)$, ACE $27>0(\mathrm{OR}=5.7$, p 0.003$)$ and $\mathrm{HCT}-\mathrm{CI}>0(\mathrm{OR}=3.87, \mathrm{p} 0.02)$ were contributing factors to 40 -day mortality, but no meaningful association was noticed with the other scales. Therefore, this study reaffirms the significant impact of comorbidities on the survival of patients with $\mathrm{AL}$, suggesting that comorbidity assessment may be extremely helpful for making decisions on intensive induction therapy.
\end{abstract}

Keywords: Acute Leukemia, Induction Chemotherapy, Outcomes, Comorbidity

\section{Introduction}

Leukemia is responsible for approximately $3 \%$ of all cancer cases in Brazil and worldwide [1]. For Brazil, 5,940 new cases are estimated in men and 4,860 in women for each year of the 2018-2019 biennium [2]. Acute leukemias (ALs) are life-threatening diseases if not quickly treated [3]. Mortality in this disease reflects, in part, its inherent resistance to therapy and, in part, the deleterious and sometimes lethal effects of treatment [4].

The decision on whether to pursue a curative approach with intensive induction chemotherapy followed by allogeneic haemopoietic stem cell transplantation (HSCT) has to be made before the start of the regimen [5]. Therapy guidelines have largely focused on an arbitrary age cut-off as a guide for intensity of therapy [4]. However, treatment outcomes for patients treated with different therapeutic approaches depend on more important prognostic factors, such as performance status (PS) and the presence of comorbidities [6].

Pretreatment factors that affect prognosis for patients with AL can be divided into those related to the patient's ability to tolerate therapy and those related to the inherent chemosensitivity or chemoresistance of the disease itself. Patient-related variables that affect a patient's ability to 
receive adequate therapy include advanced age, poor performance status, and the presence of clinically significant medical comorbidities [7, 8].

The comorbid involvement of major organs is a poor prognostic factor for worse survival [6]. It is defined by the presence of one or more chronic health conditions that are etiologically different from the primary disease, and may contribute through different mechanisms to the poor prognosis of AL patients [9]. Patients with this risk factor have higher treatment-related mortality when treated with intensive chemotherapy. Because of the possible complications of induction therapy and prolonged neutropenia, evaluation of comorbidity may yield different results, depending on the time of assessment [5-7].

Therefore, the assessment of comorbidities may better reflect biological age and may be more useful to evaluate prognosis at the time of initial diagnosis, also having a greater influence on treatment-related clinical decisions [8,9].

Given the importance of a better understanding of the impact of baseline comorbidity and PS on early mortality and long-term outcomes in AL to improve clinical decisions and reduce treatment-related mortality, this study aims to evaluate clinical scales as predictors of mortality in patients with acute leukemia during intensive induction therapy, in a reference hospital in the Amazon region.

\section{Methods}

\subsection{Study Population}

This prospective cohort study included all patients diagnosed with Acute Myeloid Leukemia (AML) or Acute Lymphoblastic Leukemia (ALL) who received induction treatment at Ophir Loyola Hospital (HOL) in Belém-PA - a reference hospital in the treatment of haematological malignancies in the Amazon region - from February 2018 to February 2019.

All participants of the study were aged 18 years or older and provided informed consent. Patients with acute promyelocytic leukemia (APL) or those receiving less intense regimens (palliative chemotherapy) were excluded from the study.

Patient follow-up began at the time of admission, with clinical interviews to apply clinical scales until a maximum of 10 days after induction therapy had started. Outcomes were assessed at subsequent visits during the period.

\subsection{Covariates}

Covariates in this analysis included sociodemographic information, clinical data, and the type and date of induction chemotherapy. The following scales were assessed in the cohort: Eastern Cooperative Oncology Group (ECOG) scale to evaluate performance status; Haematopoetic Cell Transplantation Comorbidity Index (HCT-CI) score, Cumulative Illness Rating Scale (CIRS), Charlson Comorbidity Index (CCI) and Adult Comorbidity Evaluation 27 (ACE-27) to evaluate comorbidities.

Other parameters were also collected: functionality using the Katz Index of Independence in Activities of Daily Living and Lawton Instrumental Activities of Daily Living scale; evaluation of outcomes in oncology using the G8 Questionnaire; and nutritional evaluation using the Mini Nutritional Assessment (MAN).

\subsection{Outcomes}

Outcomes were overall survival (OS) from date of induction chemotherapy initiation, and treatment response, defined as complete remission, refractoriness or 40-day mortality. Forty-day mortality was calculated from the date of initiation of induction chemotherapy. Complete remission was defined as a morphological leukemia-free state, including less than $5 \%$ blasts in the bone marrow and recovery of haematological parameters.

\subsection{Statistical Analysis}

All statistical analyses were performed with SPSS statistics ${ }^{\circledR}$ version 18. A two-sided alpha level of 0.05 was used to indicate statistical significance. Relative risks for association between covariates and the events were estimated with a $95 \%$ confidence interval (CI).

The probability of overall survival and cumulative relapse rate were evaluated by the Kaplan-Meier method. The log rank test for overall survival was employed to study the prognosis significance of each variable.

\section{Results}

The median age of the 40 patients included was 37 years old (range, 19-65) and sex distribution was equal. All patient characteristics are listed in Table 1. At the time of therapy response assessment, 55\% achieved complete remission, 20\% had refractoriness to induction therapy and 40-day mortality rate was $25 \%$.

Table 1. Patient characteristics.

\begin{tabular}{ll}
\hline \multirow{2}{*}{ Variables } & Total \\
\cline { 2 - 2 } & $\mathbf{N}=\mathbf{4 0}(\mathbf{\%})$ \\
\hline Age $(19-65)$ & $19(47.5 \%)$ \\
$>37$ & $21(52.5 \%)$ \\
Gender & \\
Male & $20(50 \%)$ \\
Female & $20(50 \%)$ \\
Marital status & $12(30 \%)$ \\
Single & $26(65 \%)$ \\
Married & $2(5 \%)$ \\
Divorced & \\
Origin & $7(17.5 \%)$ \\
Metropolitan region & $33(82.5 \%)$ \\
Non-metropolitan Region & \\
Education level & $21(52.5 \%)$ \\
Primary & $18(45 \%)$ \\
Secondary & $1(2.5 \%)$ \\
High & \\
Diagnosis &
\end{tabular}




\begin{tabular}{ll}
\hline \multirow{2}{*}{ Variables } & Total \\
\cline { 2 - 2 } & $\mathbf{N}=\mathbf{4 0}(\mathbf{\%})$ \\
\hline ALL & $19(47.5 \%)$ \\
AML & $21(52.5 \%)$ \\
Line of therapy & \\
First & $22(55 \%)$ \\
Second and further & $18(45 \%)$ \\
Treatment Response & \\
Complete Remission & $22(55 \%)$ \\
Refractoriness & $8(20 \%)$ \\
40-day mortality & $10(25 \%)$ \\
\hline
\end{tabular}

Table 2. Distribution of comorbidities and P-value of association with 40day mortality.

\begin{tabular}{|c|c|c|}
\hline \multirow{2}{*}{ Variables } & \multicolumn{2}{|l|}{ Total } \\
\hline & $\mathrm{N}=40(\%)$ & P-value \\
\hline ECOG & & 0.836 \\
\hline$>1$ & $16(40 \%)$ & \\
\hline$<2$ & $24(60 \%)$ & \\
\hline CIRS & & 0.203 \\
\hline$>5$ & $14(35 \%)$ & \\
\hline$<6$ & $26(65 \%)$ & \\
\hline HCT-CI & & 0.02 \\
\hline$>0$ & $10(25 \%)$ & \\
\hline$<1$ & $30(75 \%)$ & \\
\hline $\mathrm{CCI}$ & & 0.194 \\
\hline$>2$ & $11(2.5 \%)$ & \\
\hline$<3$ & $29(72.5 \%)$ & \\
\hline ACE-27 & & 0.003 \\
\hline$>0$ & $10(25 \%)$ & \\
\hline$<1$ & $30(75 \%)$ & \\
\hline G8 & & 0.512 \\
\hline$>14$ & $7(18.4 \%)$ & \\
\hline$<15$ & $31(81.6 \%)$ & \\
\hline MNA & & 0.683 \\
\hline$>11$ & $6(15.8 \%)$ & \\
\hline$<12$ & $32(84.2 \%)$ & \\
\hline KATZ & & 0.216 \\
\hline$>4$ & $34(85 \%)$ & \\
\hline$<5$ & $6(15 \%)$ & \\
\hline LAWTON & & 0.753 \\
\hline$>24$ & $18(45 \%)$ & \\
\hline$<25$ & $22(55 \%)$ & \\
\hline
\end{tabular}

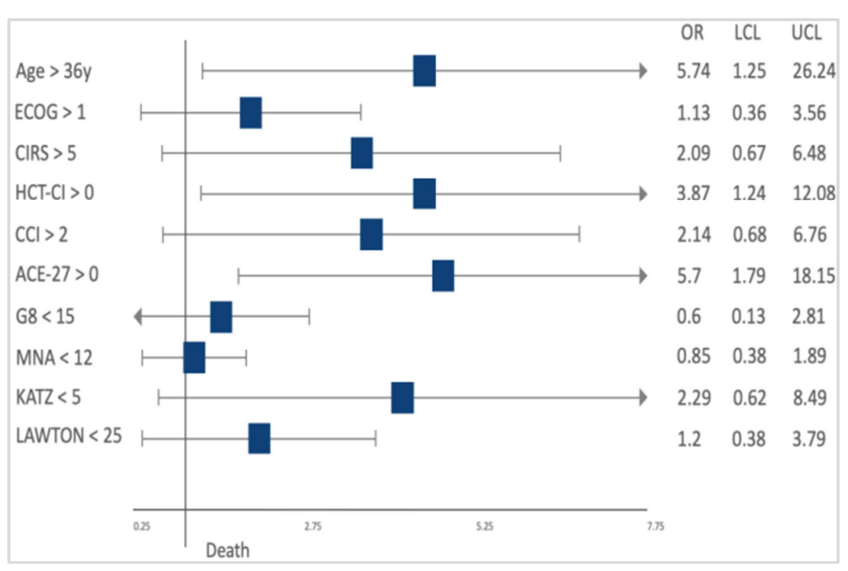

Figure 1. Univariate Cox proportional hazard regression model with factors predicting overall survival.

Univariate analysis showed that ACE $27>0(\mathrm{OR}=5.7$, p 0.003$)$ and HCT-CI $>0(\mathrm{OR}=3.87, \mathrm{p} 0.02)$ were contributing factors to 40-day mortality, but no meaningful association was noticed between CCI CIRS, ECOG PS, Katz, Lawton, MNA, G8 with induction mortality, as shown in Table 2.

Older patients ( $>36$ years) had a higher risk of death within 40 days after the initiation of induction therapy compared to younger patients ( $<36$ years), with an overall survival rate of $57.1 \% \times 89.5 \%$, respectively $(\mathrm{OR}=5.74$, p 0.024).
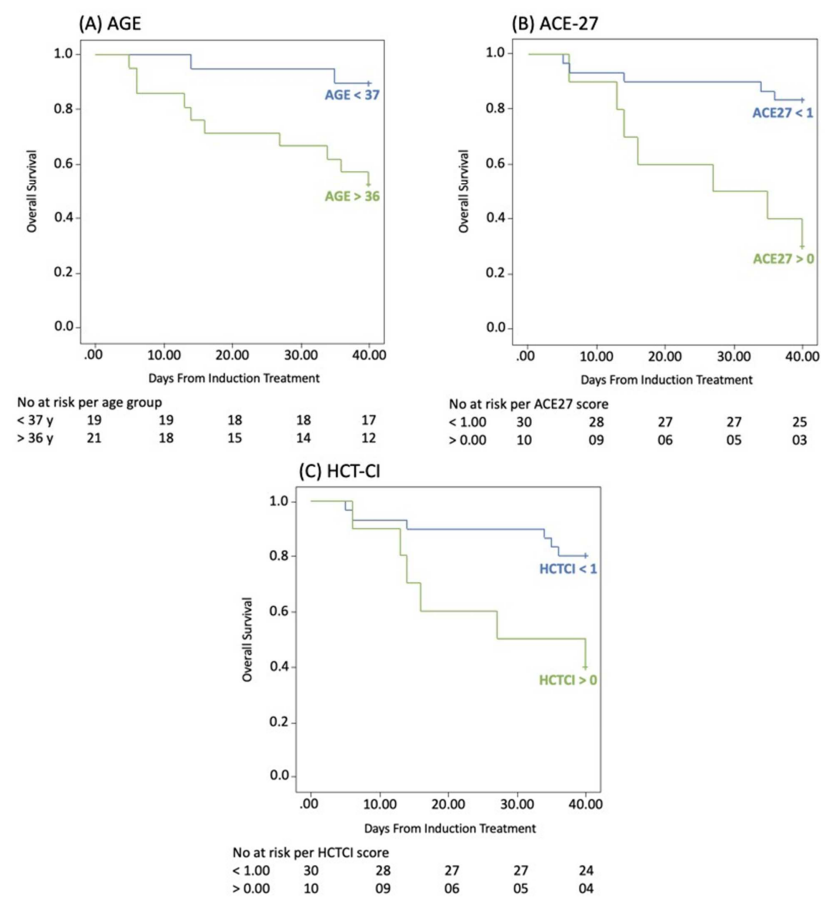

Figure 2. Kaplan-Meier Estimates of Survival according to (A) age, (B) $A C E-27$ and (C) HCT-CI.

\section{Discussion}

The measurement of comorbidity burden to predict treatment outcomes in patients with cancer has been frequently investigated [10]. In this study, univariate analysis demonstrated that higher age, ACE $27>0$ and HCT-CI $>0$ were contributing factors for 40-day mortality. The other indexes studied were not associated with worst survival.

There are reports that identify age as a risk factor for worse OS in patients with AML $[10,11]$. According to this result, Master et al. [12] found that patients with older age and a higher comorbidity index also had the worst AML survival: age-related mortality increased to approximately double in the 50-64 years group compared to 18-49 years. Risk of death was even higher in those aged 65 years and older.

A different study, on the other hand, could not demonstrate an association between age and OS in multivariate analysis when comorbidity scores were included. It concluded that the influence of age on survival is probably due to an age-related comorbidity burden [8].

ECOG performance status did not seem to have a meaningful association with 40-day mortality, which is not 
consistent with another cohorts, such as Wass et al. [8] who identified an ECOG performance status $\geq 2$ as a single independent risk factor for early death, and even reported that this score is a stronger predictor for early death than comorbidity burden.

In this study, only ACE-27 and HCT-CI demonstrated significant correlations between comorbidity and higher risk of death. Patients who had an ACE-27 $>0$ presented a 40-day OS rate of $30 \%$, compared to $83,3 \%$ of those who had ACE$27<1$, which means an approximately 6 times higher chance of death in 40 days. Likewise, a study that analysed patients with head and neck cancer showed a dose-response relationship between comorbidity collected by ACE-27 and survival [13].

Previous reports using HCT-CI to determine the impact of comorbidities on the survival of AML patients also concluded that it is a significant predictor of early death $[4,8$, 11]. In keeping with previous research, our baseline data confirmed the association. HCT-CI $>0$ represented a 4 times higher chance of 40-day mortality, with an OS rate of $40 \%$, in contrast with an OS rate of $80 \%$ in the HCT-CI $<1$ group.

In a retrospective cohort study, HCT-CI, ACE-27 and CIRS-G were applied to analyse the association between indices-based comorbidity scores with AML patient outcome, but, in multivariate analysis, only the ACE-27 score was associated with outcomes [8].

Ito et al. [14] demonstrated that a CCI score of less than 2 points correlated with a favourable clinical outcome in cancer patients aged over 75 years. This was not reproduced in our younger aged cohort.

These findings may be due to the fact that ACE-27 covers a wider range of comorbidities in comparison to $\mathrm{CCI}$, identifying different number, types, and severity of comorbidities. [15]. Furthermore, CCI seems to be more subjective than other scales such as HCT-CI [6].

Nutritional status is another condition that is widely associated with the prognosis of AL patients. Li et al. [16] suggested that severely malnourished patients are more likely to have side effects of chemotherapy and, therefore, shorter survival time. Similarly, Baumgartner et al. [17] demonstrated that low initial body mass index and more pronounced weight loss were strong indicators for lower survival and worse disease outcomes in their study with AML patients.

However, nutritional status, measured by MNA and G8, did not present a relationship to clinical outcomes in our study. That may be due to the fact that this study does not have a significant number of malnourished patients.

Besides, although an evaluation of functional capacity can allow an understanding of factors that go beyond chronological age, such as expectation and quality of life, risks and benefits of treatments, patients functionality, evaluated by Katz and Lawton scales, were not associated with any outcomes in this study. That finding may have occurred because patients in this study were younger compared to those in which those functionality scales were applied [18].
This study has several limitations. The sample size was small, limiting the detection of differences in assessment measures: small differences in comorbidity scores may not achieve significance in a sample of this size. Also, this is a single institution study which may limit the generalisability of findings.

However, the inclusion of only those patients receiving intensive therapy and the prospective character of the study minimised the confounding effects of treatment on comorbidity: longitudinal changes could be observed over time.

\section{Conclusion}

Despite the study limitations, such as small sample size, the prospective character of the study allowed it to observe the same patients and their changes over time. Higher age, ACE $27>0$ and HCT-CI $>0$ were shown to have a positive association with 40 -day mortality, which reaffirms the significant impact of advanced age and comorbidities on patient's ability to receive adequate therapy for AL and their survival. Although further study with a larger number of cases in a multi-institutional setting is required, these findings support the need of comorbidity assessment through clinical scales for making decisions on intensive induction therapy and to provide more accurate survival estimates.

\section{References}

[1] Rêgo MAV, Fonseca AA. Mortality Trends from Leukemia in Salvador - Brazil, 1980 to 2012. Rev Bras Cancerol. 2015; 61 (4): 325-34.

[2] Instituto Nacional de Câncer José Alencar Gomes da Silva. Estimate 2018: incidence of cancer in Brazil / Instituto Nacional de Câncer José Alencar Gomes da Silva. Coordenação de Prevenção e Vigilância. - Rio de Janeiro: INCA, 2017.

[3] Righes CS, Garcia DVO, Silva PC, Almeida EB, Pellegrin JC. Epidemiological, hematological and imunophenotypic evaluation of adult patients with acute leukemia diagnosed at the Regional Hospital of Mato Grosso do Sul, Brazil. RBAC. 2017; 49 (3): 249-55.

[4] Sorror ML, Storer BE, Fathi AT, et al. Development And Validation Of A Novel Acute Myeloid Leukemia-Composite Model To Estimate Risks Of Mortality. JAMA Oncol. 2017; $1-8$.

[5] Middeke JM, Herbst R, Parmentier S, et al. Long-Term Follow-Up and Impact of Comorbidity before Allogeneic Hematopoietic Stem Cell Transplantation in Patients with Relapsed or Refractory Acute Myeloid Leukemia-Lessons Learned from the Prospective BRIDGE Trial. Biol Blood Marrow Transplant. 2017; 23: 1491-1497.

[6] Budziszewska BK, Pluta A, Sulek K, et al. Treatment of elderly patients with acute myeloid leukemia adjusted for performance status and presence of comorbidities: a Polish Adult Leukemia Group study. Leuk \& Lymphoma. 2015; 1-8. 
[7] Short, NJ, Rytting, ME, Cortes, JE. Acute myeloid leukaemia. Lancet 2018, 392, 593-606.

[8] Wass M, Hitz F, Schaffrath J, MullerTidow C, Muller LP. Value of Different Comorbidity Indices for Predicting Outcome in Patients with Acute Myeloid Leukemia. PLoS One. 2016; 11 (10): 1-13.

[9] Dufva IH, GranfeldtØstg ard LS, Medeiros BC, et al. Epidemiology and clinical significance of secondary and therapy-related acute myeloid leukemia: a national populationbased cohort study. J Clin Oncol. 2015; 33 (31): 3641-3649.

[10] Tawfik B, Pardee TS, Isom S, et al. Comorbidity, age, and mortality among adults treated intensively for acute myeloid leukemia (AML), J Geriatr Oncol. (2015), http://dx.doi.org/10.1016/j.jgo.2015.10.182

[11] Djunic I, Virijevic M, Novkovic A, et al. Pretreatment risk factors and importance of comorbidity for overall survival, complete remission, and early death in patients with acute myeloid leukemia. Hematology. 2012; 17(2): 53-38.

[12] Master S, Munker R, Shi Z, Mills G, Shi R. Insurance Status and Other Non-biological Factors Predict Outcomes in Acute Myelogenous Leukemia: Analysis of Data from the National Cancer Database. Anticancer research. 2016; 36: 4915-22.
[13] Schimansky S, Lang S, Beynon R, et al. Association between comorbidity and survival in head and neck cancer: Results from Head and Neck 5000. Head \& Neck. 2018; 1-10.

[14] Ito S, Ito $\mathrm{H}$, Sato $\mathrm{N}$, et al. Clinical factors associated with the therapeutic outcome of chemotherapy in very elderly cancer patients. Int J Clin Oncol. 2019. https://doi.org/10.1007/s10147-018-01385-8

[15] Kallogjeri D, Gaynor SM, Piccirillo ML, et al. Comparison of Comorbidity Collection Methods. J Am Coll Surg. 2014; 219 (2): 245-255.

[16] Li J, Wang C, Liu X, et al. Severe malnutrition evaluated by patient-generated subjective global assessment results in poor outcome among adult patients with acute leukemia: A retrospective cohort study. Medicine. 2018; 97 (3): 1-6.

[17] Baumgartner A, Zueger N, Bargetzi A, et al. Association of Nutritional Parameters with Clinical Outcomes in Patients with Acute Myeloid Leukemia Undergoing Haematopoietic Stem Cell Transplantation. Ann Nutr Metab. 2016; 69: 89-98.

[18] Pereira EEB, Sarges ESNF, Santos NB. Functional capacity evaluation of the hospitalized oncogeriatric patient. Rev PanAmaz Saude. 2014; 5 (4): 37-44. 